\title{
Tuning the Balance between Fibrillation and Oligomerization of $\alpha$-Synuclein in the Presence of Dopamine
}

\author{
Shivnetra Saha, Mohammad Ashhar Iqbal Khan, ${ }^{\dagger}$ Deepak Mudhara, and Shashank Deep*(i) \\ Department of Chemistry, Indian Institute of Technology Delhi, Hauz Khas, New Delhi 110016, India \\ Supporting Information
}

ABSTRACT: The aggregates of $\alpha$-synuclein bear a close connection with Parkinson's disease, which is largely characterized by the loss of the dopaminergic neurons. Dopamine promotes the formation of undesirable sodium dodecyl sulfate (SDS)-resistant oligomers of $\alpha$-synuclein. In this study, we have shown that the inhibition of fibrillation by an additive may not always be the ultimate deciding factor in the context of its potential as a successful additive. Copper promotes the fibrillation of $\alpha$-synuclein in buffer alone but inhibits the formation of SDS-resistant oligomers in the presence of dopamine. Glycerol, on the other hand, increases the population of such dopamine-mediated SDSresistant oligomers. We speculate such an effect to be a manifestation of the distinct oxidation pathway of dopamine in the presence of copper.

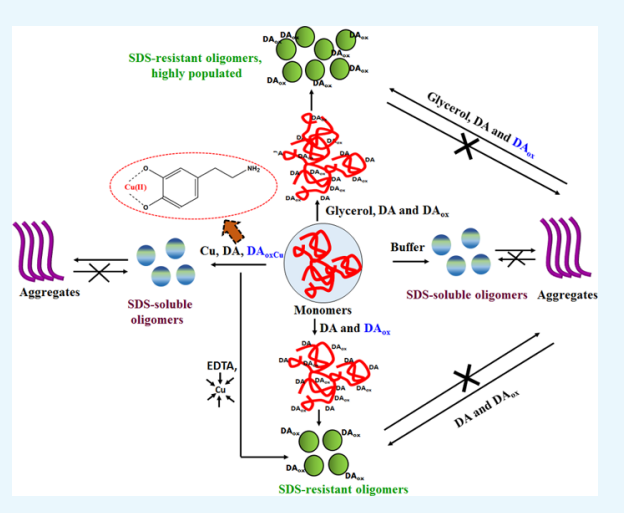

\section{INTRODUCTION}

Parkinson's disease (PD), a debilitating neurodegenerative disease, is associated with the impairment of motor neurons such as bradykinesia, resting tremors, and rigidity. ${ }^{1}$ Ordered aggregates of proteins, more commonly known as the amyloid fibrils, have been found to be a major contributor in this and many other neurodegenerative diseases such as Alzheimer's, Huntington's, and Amyotrophic lateral sclerosis. A plethora of studies have shown that it is not these fibrils, but the precursors of these fibrils, that is, the prefibrillar aggregates and the oligomers, that are toxic to the cellular system.

The symptoms of PD are attributed to the loss of the dopaminergic neurons [the main source of dopamine (DA)] in the substantia nigra of the midbrain. DA is an important neurotransmitter in the physiological system which sends signals to other nerve cells and plays a significant role in the motor movements, release of various hormones, and rewardmotivated behavior. DA is structurally a catecholamine having a very low oxidation potential. Thus, it is highly prone to oxidation and is unstable under the cytosolic environment. Its oxidation leads to the generation of reactive oxygen species such as superoxide, hydrogen peroxide, and also DA-reactive dopaminoquinones and dopaminochromes, which prove toxic to the cellular system. ${ }^{2-6}$ Given the sensitivity of DA to oxidation, it is believed that DA oxidation must have a major contribution to the disease.

The neurons in the substantia nigra of the midbrain region of PD patients contain Lewy bodies, which are essentially the aggregates of a small, intrinsically disordered protein, $\alpha$ synuclein. ${ }^{4,8}$ It has been found that the dopaminochrome formed as a result of the autooxidation of cytosolic DA forms a covalent adduct with $\alpha$-synuclein and promotes the formation of a specific type of oligomers-those that are resistant to sodium dodecyl sulfate (SDS). ${ }^{3,5,6,9,10}$ DA stabilizes the oligomers and prevents the further growth of these oligomers into mature fibrils. These SDS-resistant oligomers are responsible for imparting toxicity to the physiological system. $^{2,3}$ The oligomers in DA are in contrast to the commonly observed oligomers which are soluble in SDS. The effect of DA is so strong that it can even interact with the fibrils to disintegrate them into the SDS-resistant oligomers. In addition, these SDS-resistant oligomers formed in DA are found to be more toxic than the oligomers formed in the absence of DA. A plethora of studies exists, which have examined the mechanism of the action of DA on $\alpha$-synuclein and its aggregation. In this study, however, we have made an attempt to assess the factors which can modulate the population of these SDS-resistant oligomers formed in DA.

Decoding the routes to modulate the toxic species remains an integral part in the therapeutics of many diseases, and a broad spectrum of studies exist, which have used external additives to do so. Additives ranging from osmolytes, polyphenols, small organic compounds, metal ions, pesticides, and nanoparticles have been tested on the different aspects of proteins such as their stability, activity, and aggregation. Here, we have examined the effect of a metal ion, copper, and an osmolyte, glycerol, on the aggregation of $\alpha$-synuclein in the presence of DA. Copper, in particular, is a well-known enhancer of the fibrillation of $\alpha$-synuclein, which initiates the process by binding to the monomeric protein. ${ }^{11-14}$ On the other hand, glycerol has been long known to inhibit or suppress the aggregation of a number of proteins. ${ }^{15-18}$ The

Received: May 14, 2018

Accepted: October 2, 2018

Published: October 26, 2018 

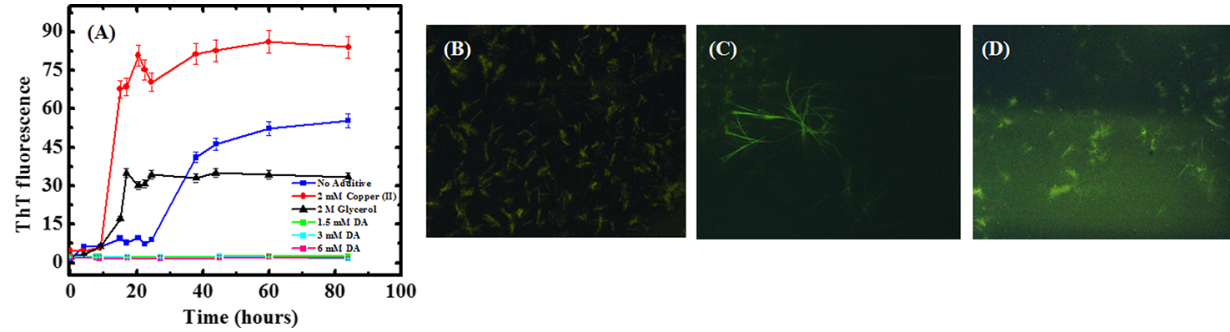

Figure 1. (A) Aggregation of $35 \mu \mathrm{M} \alpha$-synuclein in $20 \mathrm{mM}$ Tris buffer, $0.1 \mathrm{M} \mathrm{NaCl}$ (pH 7.5), in the presence and absence of different additives at $37^{\circ} \mathrm{C}, 250 \mathrm{rpm}$, monitored by ThT fluorescence. Fluorescence microscopy images of the aggregates of $\alpha$-synuclein formed (B) in the absence of any additive, (C) in the presence of copper, and (D) in the presence of glycerol.
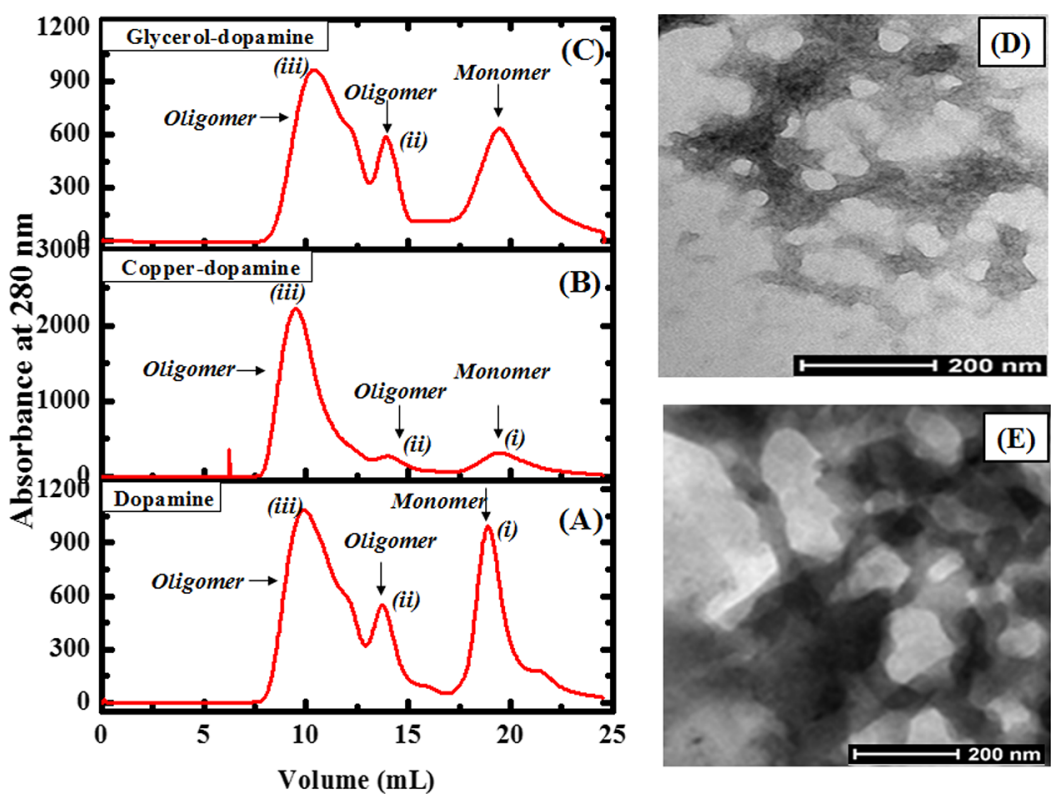

Figure 2. Size exclusion profiles of $200 \mu \mathrm{M} \alpha$-synuclein after its aggregation in DA (A) without any additive (B) with copper and (C) with glycerol. TEM images of the aggregates of $\alpha$-synuclein formed in DA in the presence of (D) copper and (E) glycerol.

origin of the choice of additives was aimed with the idea to investigate the effect of an aggregation enhancer and a suppressor on the population of these DA-mediated SDSresistant oligomers. A wide range of studies exist, which have individually explored the effect of different additives or DA on the aggregation of $\alpha$-synuclein. However, to the best of our knowledge, not many studies have been carried out on a combined behavior of the additives and DA on the protein-a scenario which would resemble the physiological system more closely.

Interestingly, our study shows that copper (generally an enhancer of fibrillation) mitigates the formation of the SDSresistant oligomers which are formed in the presence of DA, whereas glycerol (generally an inhibitor of fibrillation), on the other hand, promotes the formation of these DA-mediated oligomers. This is in contrast to the behavior of copper or glycerol in the absence of DA where they are found to enhance or inhibit, respectively, the oligomerization and fibrillation. Our analysis suggests that the kinetics and the nature of the chemical interaction of copper with DA modify the later in such a way that it is not able to interact with the protein. The absence of this interaction allows the aggregation to proceed further, thus decreasing the population of the SDS-resistant oligomers.

\section{RESULTS AND DISCUSSION}

Glycerol Inhibits Fibrillation of $\alpha$-Synuclein in the Absence of DA. The effect of copper and glycerol on the fibrillation of $\alpha$-synuclein in the absence of DA was monitored by thioflavin $\mathrm{T}$ (ThT), which is capable of binding to fibrils. Despite the fact that both copper and glycerol accelerated the onset of fibrillation, the former enhanced the extent of fibrillation, whereas the latter reduced the same (Figure 1A). Fluorescence microscopy of the aggregates also showed the presence of fibrils in both the additives, although fewer fibrils could be seen in glycerol than in copper or buffer alone (Figure $1 \mathrm{~B}-\mathrm{D})$, which was in sync with the reduced ThT fluorescence in glycerol. This suggested that glycerol could be an effective inhibitor of the fibrillation of $\alpha$-synuclein in the absence of DA. The decrease in the extent of fibrillation by glycerol may not be unexpected in the first place as it has been widely proven as an aggregation suppressor and a promoter of the folded state of the protein. In fact, glycerol has also been found to rectify the misfolded conformations of the protein inside the endoplasmic reticulum, ${ }^{19,20}$ in which case, it is worth exploring for a positive role under in vivo conditions. Aggregation in the presence of DA, however, did not show any change in the ThT fluorescence (Figure 1A), and this phenomenon is well documented in the literature. ${ }^{21}$ 
(A)

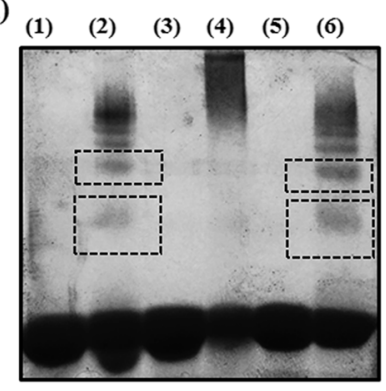

(B)

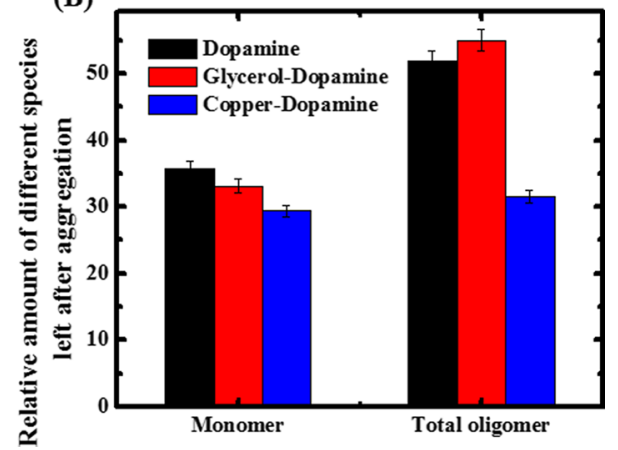

Figure 3. (A): SDS-PAGE of the aggregates of $\alpha$-synuclein formed in buffer alone (lane 1), copper (lane 3), glycerol (lane 5), DA (lane 2), DA and copper (lane 4), and DA and glycerol (lane 6). (B): Quantification of monomers and oligomers from the SDS-PAGE in panel A.
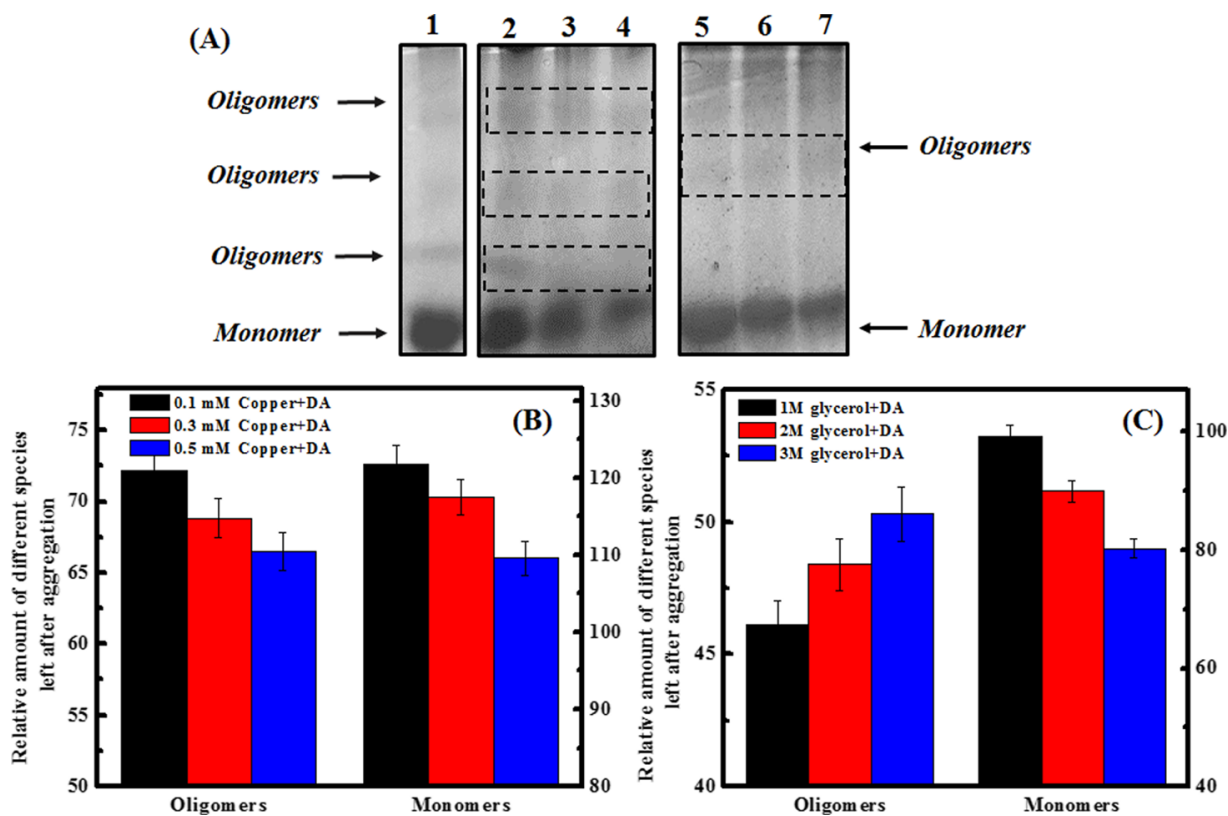

Figure 4. (A) SDS-PAGE analysis of the aggregation of $200 \mu \mathrm{M} \alpha$-synuclein in the presence of DA alone (lane 1 ), in the presence DA and $0.1 \mathrm{mM}$ copper (lane 2), $0.3 \mathrm{mM}$ copper (lane 3), $0.5 \mathrm{mM}$ copper (lane 4), $1 \mathrm{M}$ glycerol (lane 5), $2 \mathrm{M}$ glycerol (lane 6), and $3 \mathrm{M}$ glycerol (lane 7). Quantification of the monomers and oligomers detected in SDS-PAGE at the end of aggregation of $\alpha$-synuclein in DA in the presence of copper (B) and glycerol (C).

Association States of $\alpha$-Synuclein in DA in the Presence of Copper and Glycerol. Because of the absence of any ThT fluorescence in the presence of DA, the various species populated under the conditions of aggregation were studied using size exclusion chromatography. The size exclusion chromatograms of $\alpha$-synuclein aggregated in the presence of DA showed a significant presence of oligomers around elution volume of 9.9 and $13 \mathrm{~mL}$ (Figure 2A), indicating that although no ThT fluorescence could be detected, the protein did associate into higher-order species in the presence of DA. A combination of copper and DA (copper-DA) was found to increase the population of the oligomers eluting around $9.9 \mathrm{~mL}$, whereas it decreased the population of the oligomers eluting around $13 \mathrm{~mL}$ (Figure 2B). The population of monomers was also greatly reduced in copper-DA, suggesting that copper could deplete the monomers from the solution with a much faster kinetics. In the presence of glycerol and DA (glycerol-DA), the population of oligomers around $13 \mathrm{~mL}$ was similar, whereas that around $9.9 \mathrm{~mL}$ was slightly higher than that in $\mathrm{DA}$ alone
(Figure 2C). The monomer content remaining in the glycerol-DA system was considerably low as compared to that in DA alone. The transmission electron microscopy (TEM) images of the aggregates in copper-DA and glycerolDA exhibit a wormlike morphology, as shown in Figure 2D,E.

Oligomers Formed in Copper-DA Are Soluble in SDS, Whereas Those Formed in DA or Glycerol-DA Are Resistant to SDS. Studies have shown that a catechol moiety (which is also present in DA) is essential for the inhibition of fibrillation and promotion of SDS-resistant oligomers in $\alpha$ synuclein. ${ }^{5,9}$ The most convenient technique of detecting these SDS-resistant oligomers is SDS polyacrylamide gel electrophoresis (PAGE). The aggregation of $\alpha$-synuclein in buffer alone, copper, and glycerol, in the absence of DA did not show the presence of any oligomers in SDS-PAGE but showed a thick band corresponding to the monomer (Figure 3A, lanes 1, 3 , and 5). It is already known that the aggregation of $\alpha$ synuclein in the absence of DA proceeds via the formation of oligomers, but the absence of any oligomers in SDS-PAGE showed that such oligomers are strictly the ones that are 
soluble in SDS. However, oligomeric species could be clearly detected in SDS-PAGE when $\alpha$-synuclein was aggregated in the presence of DA (Figure 3A, lane 2). It is also evident from the figure that the oligomers were more predominant in the aggregation in the presence of glycerol and DA, suggesting that glycerol increases the formation of SDS-resistant oligomers (Figure 3A, lane 6).

Surprisingly, only a minute amount of oligomers could be seen in the presence of copper and DA (Figure 3A, lane 4), suggesting that copper did not promote or stabilize the SDSresistant oligomers. In order to get a more confirmatory conclusion, the bands in the gels were quantified. The analysis clearly suggests that the population of the SDS-resistant oligomers decreased by about $64 \%$ in the presence of copper and DA, whereas they increased by nearly $8 \%$ in the presence of glycerol and DA, as compared to that in DA alone (Figure 3B).

The effect of copper and glycerol was further confirmed by aggregating $\alpha$-synuclein in the presence of DA, with different concentrations of copper and glycerol. It could be seen that increasing concentrations of copper gradually decreased the formation of SDS-resistant oligomers (Figure 4A). Subsequently, the residual monomer in the presence of $0.5 \mathrm{mM}$ copper was also slightly less, suggesting that although monomers are consumed, they are incorporated into the insoluble aggregates, and not the SDS-resistant oligomers. The scenario was reversed in the glycerol-DA system, and the amount of SDS-resistant oligomers increased at higher glycerol concentrations (Figure 4A). Consequently, the amount of monomers left at the end of aggregation also decreased with increasing concentrations of glycerol. This demonstrates that glycerol (in the presence of DA) shifts the aggregation toward the SDS-resistant oligomers from the monomers. The relative amounts of monomers left or the oligomers formed have been quantified from the gel images and correlate well with the visual inspection (Figure 4B,C). The oligomers formed in the presence of DA alone or glycerol-DA as seen in size exclusion chromatography (Figure 2) were relatively lower than that formed in copper-DA. This is in contrast to that observed in SDS-PAGE. We reach an important conclusion here. The oligomers formed in the copper-DA system are different from those formed in the presence of DA alone or in the glycerolDA system. Although the oligomers formed in copper-DA can be disintegrated by SDS, those formed in DA alone or glycerol-DA are built up of interactions that cannot be disrupted by SDS. That is to say, the oligomers are perhaps chemically different.

Oligomers Formed in Copper-DA Are Structurally Different from Those Formed in DA Alone or in Glycerol-DA. Previous investigations have shown that the oligomers of $\alpha$-synuclein formed in buffer alone have a beta sheet structure. ${ }^{2,22}$ On the contrary, oligomers formed in DA are essentially unfolded. ${ }^{2}$ Interestingly, it was found that the oligomers formed in copper-DA have a beta sheet structure (similar to that found in the absence of DA), whereas those formed in glycerol-DA were unfolded in nature (Figure 5). This again suggests that copper can nullify the presence of DA all together, as DA does not seem to have any impact on the aggregation when copper is present. The behavior in copperDA is the same as that observed in buffer alone, in the absence of DA. This holds well both in terms of the effect of SDS on the oligomers and also the structure of the oligomers.

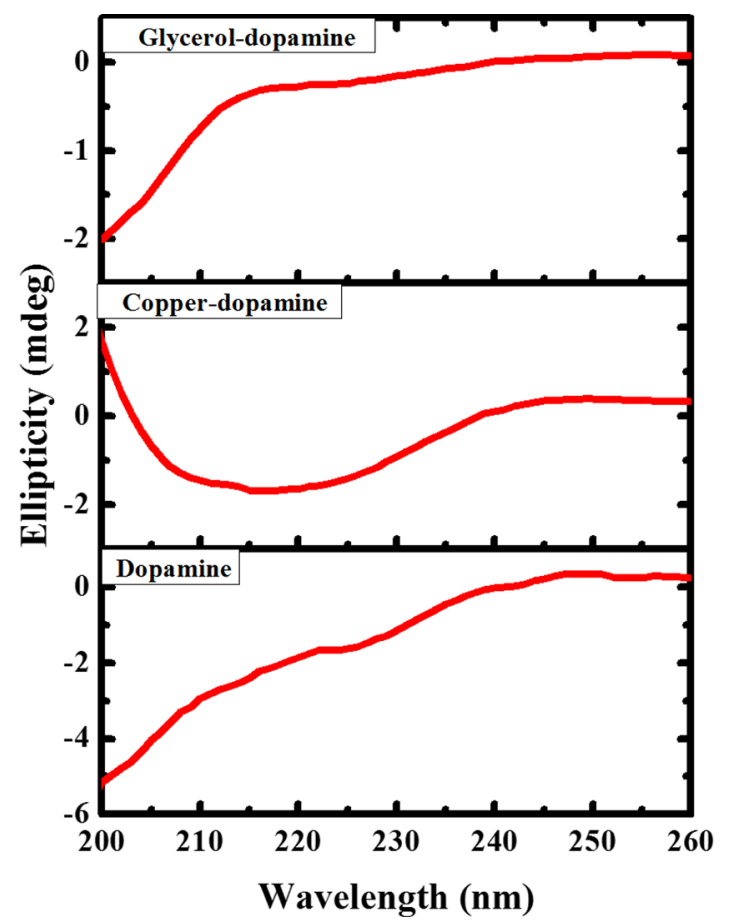

Figure 5. CD spectra of the oligomers remaining after the aggregation of $\alpha$-synuclein at $37^{\circ} \mathrm{C}, 250 \mathrm{rpm}$, in the presence of different additives.

Glycerol Increases the Population of $\alpha$-Synuclein-DA Adduct, whereas Copper Completely Suppresses the Adduct Formation. Conway et al. $^{5}$ had reported that an adduct of monomeric $\alpha$-synuclein with DA shows a unique fluorescence at $450 \mathrm{~nm}$ upon excitation at $360 \mathrm{~nm}$. Such was also true in our case (Figure 6A, red curve). The natively unfolded protein exposes a number of residues such as lysine, methionine, and tyrosine, which are capable of interacting with DA and its oxidation products (Scheme 1). On the basis of these possibilities, the observed fluorescence was attributed to a covalent, tyrosine-derived radical coupling adduct. ${ }^{5}$ The adduct can be ascribed to be formed from the hydroxyl radical in tyrosine and the hydroxyl radical in DA. That the fluorescence was a consequence of the interaction of DA with the protein was further confirmed by the fact that freshly prepared DA in buffer alone did not show any fluorescence in this region (Figure 6A, black curve) in the absence of protein. It has been shown that a derivative of DA covalently binds to $5-10 \%$ of $\alpha$-synuclein, which inhibits the further fibrillation of the remaining protein, and promotes SDS-resistant oligomerization instead. Even otherwise, the protein-DA adduct is formed only with a certain fraction of the $\alpha$-synuclein present in the medium. ${ }^{5,23}$ Our study shows that the fluorescence from the protein-DA adduct is largely enhanced in the presence of glycerol, suggesting a larger population of the adduct in the presence of this additive (Figure 6A, blue curve). Interestingly, the fluorescence was completely lost in the system containing protein, DA and copper, which meant that the protein-DA adduct was not formed at all in the presence of this metal (Figure 6A, green curve). The enhanced adduct fluorescence in glycerol-DA and the absence of any fluorescence in copperDA might hold the clue to the increased and the suppressed formation of the SDS-resistant oligomers in glycerol and copper, respectively. The absence of any adduct in the copper-DA system (unlike that in DA alone or glycerol- 

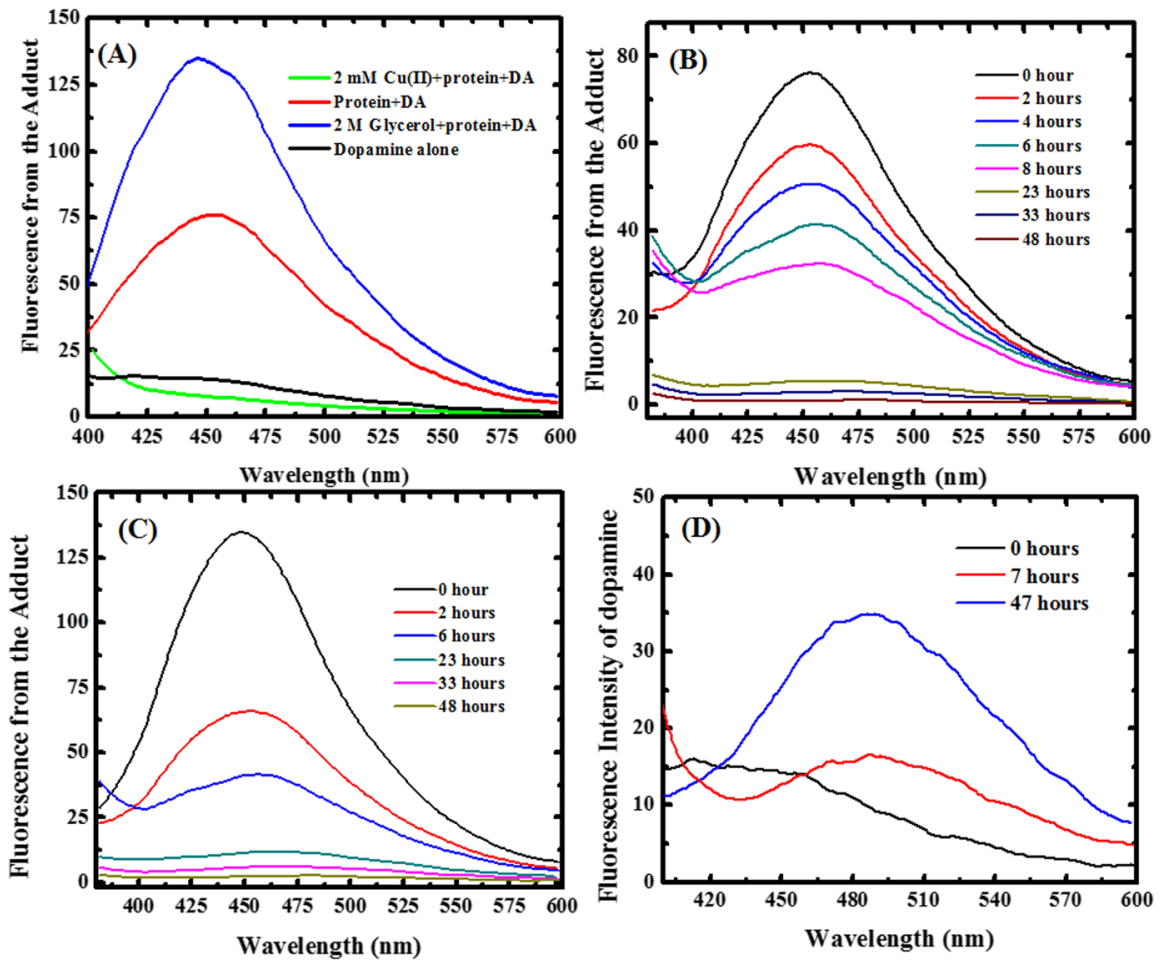

Figure 6. (A) Fluorescence spectra of the protein-DA adduct in the presence of different additives. Fluorescence spectra of DA during the aggregation of $35 \mu \mathrm{M} \alpha$-synuclein in $20 \mathrm{mM}$ Tris buffer, $0.1 \mathrm{M} \mathrm{NaCl}(\mathrm{pH} 7.5)$ at $37^{\circ} \mathrm{C}, 250 \mathrm{rpm}$, at different time points in the (B) absence and (C) presence of glycerol. (D) Fluorescence spectra of DA in buffer at different time points without any additives.

Scheme 1. Different Species in the Oxidation Pathway of DA<smiles>CCOc1ccc(CCN)cc1OC</smiles><smiles>NCCC1=CC(=O)C(=O)C=C1</smiles><smiles>Oc1cc2c(cc1O)NCC2</smiles>

Dopamine Dopamine o-semiquinone Dopaminoquinone Leukoaminochrome

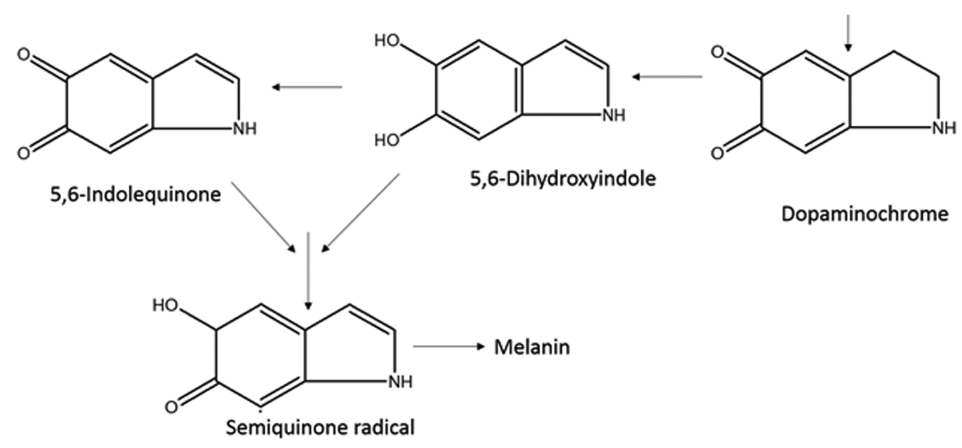

DA system) suggests a different starting point and hence a different pathway of aggregation of $\alpha$-synuclein in this system. It was due to this distinct pathway that the formation of stable SDS-resistant oligomers did not take place in the copper-DA system.

Freshly prepared DA (in buffer alone) did not show any remarkable change in the fluorescence signal with increasing time at shorter time scales (data not shown). However, the fluorescence from the protein-DA adduct under the conditions of aggregation $\left(37^{\circ} \mathrm{C}, 250 \mathrm{rpm}\right)$ showed a concomitant decrease with increasing time (Figure 6B). Size exclusion profiles and SDS-PAGE studies have already confirmed that oligomers are populated under this condition. Thus, this decrease of the adduct fluorescence can be considered to be a direct consequence of the formation of the DA-bound SDS-resistant oligomers. The rate of decrease was found to be much faster in the presence of glycerol (Figure 6C), which perhaps corresponds to a greater extent of oligomerization at a particular time point. This is also in good agreement with our SDS-PAGE results, which show that glycerol promotes the formation of the SDS-resistant oligomers. Our speculation about the decreasing intensity of the adduct might be supported by the fact that pure $\alpha$ synuclein at $25{ }^{\circ} \mathrm{C}$ incubated under quiescent conditions in the presence of DA does not show any remarkable change in the fluorescence (Figure S1).

That the decrease in the fluorescence of the adduct was indeed due to the interaction of DA and $\alpha$-synuclein oligomers 

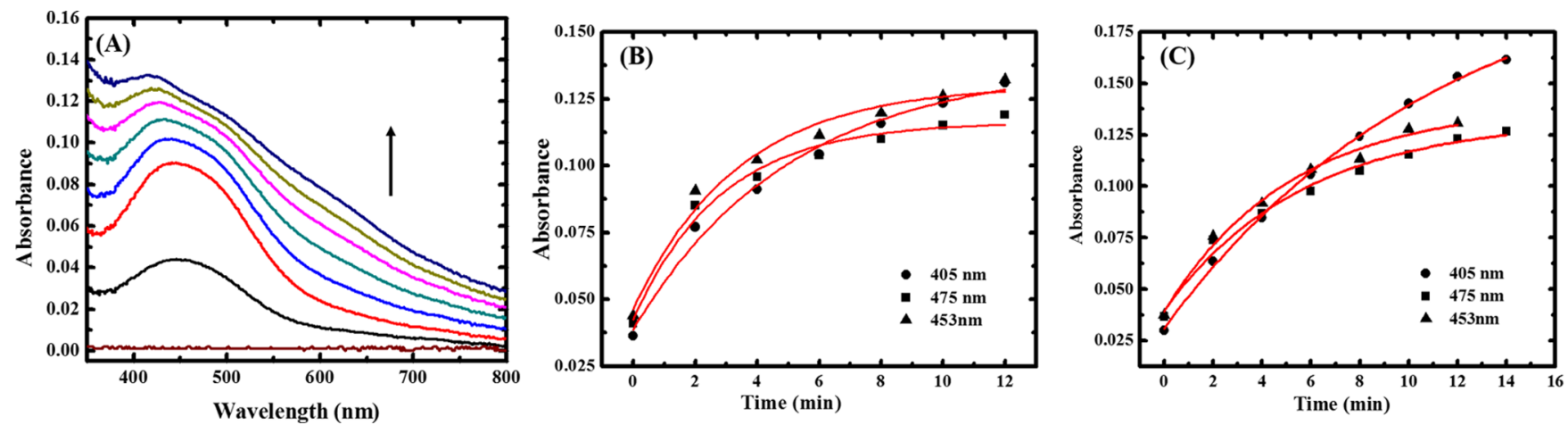

Figure 7. (A) UV absorbance spectra of DA with increasing time in buffer $(20 \mathrm{mM}$ Tris, $0.1 \mathrm{M} \mathrm{NaCl}, \mathrm{pH} 7.5)$. The arrow points toward increasing time. Change in the absorbance of the DA solution at different wavelengths with increasing time in the absence (B) and presence (C) of $35 \mu \mathrm{M} \alpha$ synuclein. Continuous red lines in $(\mathrm{B}, \mathrm{C})$ are the exponential fits to the data points.
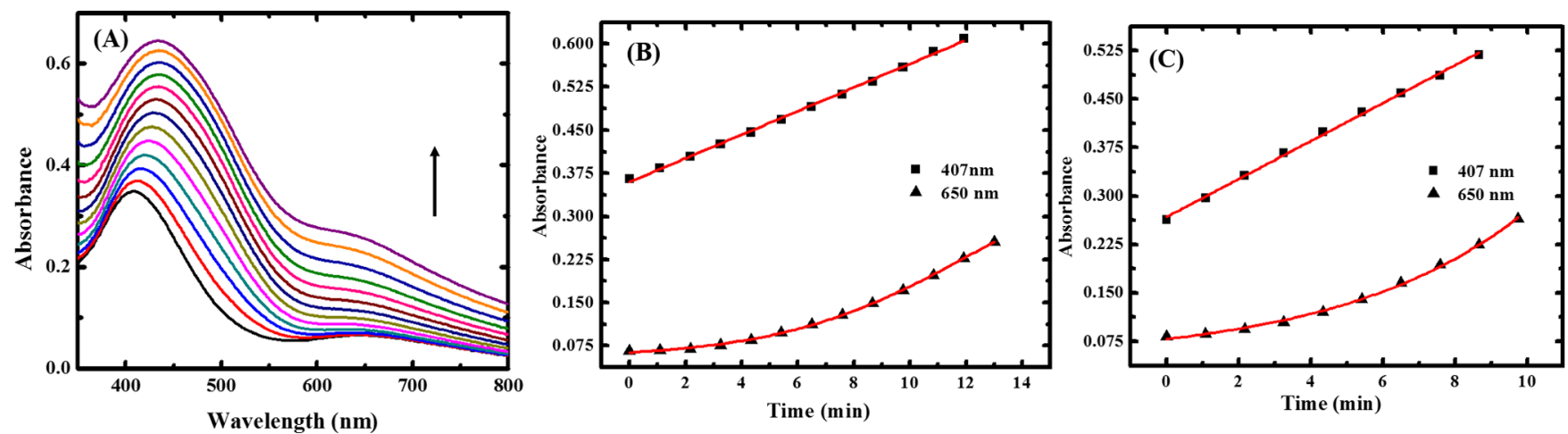

Figure 8. (A) Absorbance spectra of DA in the presence of copper in buffer ( $20 \mathrm{mM}$ Tris, $0.1 \mathrm{M} \mathrm{NaCl}, \mathrm{pH} 7.5)$. The arrow points toward increasing time. Change in the absorbance of the DA-copper system with increasing time at different wavelengths in the absence (B) and presence (C) of $35 \mu \mathrm{M} \alpha$-synuclein. Continuous red lines in (B,C) through the squares are the linear fits, and through the triangles are the exponential fits to the data points.

was completely ascertained from the fact that prolonged incubation of DA (for $47 \mathrm{~h}$ ) in buffer leads to a sharp increase in its fluorescence intensity, with the fluorescence maxima around $490 \mathrm{~nm}$ (Figure 6D). This can be attributed to the formation of 5,6-dihydroxyindole, which is the resultant soluble product of DA oxidation. ${ }^{3}$ No corresponding increase in fluorescence could be seen in the aggregating solution containing DA and the protein, suggesting that the oxidation products formed in DA do interact with $\alpha$-synuclein, resulting in species which are incapable of fluorescing.

Oxidation of DA in Buffer/Glycerol Exhibits Exponential Kinetics. The oxidation of DA at $25{ }^{\circ} \mathrm{C}$ was monitored using UV-visible spectroscopy. The absorbance spectra of DA ( $2 \mathrm{mM})$ upon immediate dissolution in buffer did not show any significant spectrum in the visible region. However, with increasing time, a prominent broad absorption band at around $453 \mathrm{~nm}$ could be seen emerging (Figure 7A). With increasing time, the intensity of absorption increased remarkably. This was also accompanied by an increased intensity of the baseline and a significant change in the shape of the spectrum, which almost lost its signature at longer times. The emergence of a distinct peak, blue-shifted toward $405 \mathrm{~nm}$, a small shoulder region red-shifted toward $475 \mathrm{~nm}$, and the loss of signature of the spectra with increasing time clearly pointed toward the formation of new products in the system. The survey of the literature suggests that the shoulder around $475 \mathrm{~nm}$ is due to the formation of dopaminochrome, ${ }^{2,24,25}$ whereas the peak observed close to $405 \mathrm{~nm}$ is due to the formation of dimers, which ultimately leads to the formation of insoluble melanin. This is reasonable because the emergence of the new peak around $405 \mathrm{~nm}$ occurs later during the reaction and correlates with the increase in scattering from the solution, indicated by an increased intensity of the baseline.

In order to account for the formation of a range of oxidation products, the absorbance spectra were analyzed at three different wavelengths of 405, 453, and $475 \mathrm{~nm}$. The kinetics of oxidation was exponential at all the three wavelengths, with minute changes in their rate (Figure $7 \mathrm{~B}$ ). The deconvolution of the spectra could not be successful because of the complexity of the curves, because of which we resorted to the analysis at multiple wavelengths. The overall signature of the spectra of the oxidation products did not change in the presence of $\alpha$-synuclein (data not shown). The kinetics was still exponential (Figure 7C), although $\alpha$-synuclein was found to retard the overall oxidation of DA. This could be attributed to a possible interaction of the oxidation products with the monomeric protein. Besides, no precipitation of DA occurred in the presence of $\alpha$-synuclein, whereas DA alone formed an insoluble precipitate in the same time scale, suggesting that the monomeric protein also interacts with the oxidation products of DA (Figure S2). Very similar trends were observed in glycerol too (Figure S3). However, the oxidation of DA in the presence of glycerol was slower than that in its absence. Additionally, the presence of protein further reduced the rate of DA oxidation in glycerol. Such inhibition (or enhancement) of the kinetics is usually believed to occur because of an 
Scheme 2. Modulation in the DA-Mediated Aggregation of $\alpha$-Synuclein by Copper

\begin{tabular}{|c|c|}
\hline Absence of Copper & Presence of Copper \\
\hline 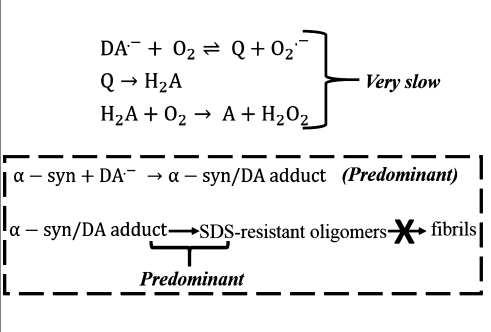 & 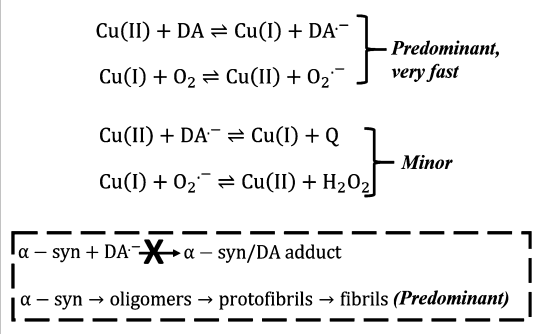 \\
\hline
\end{tabular}
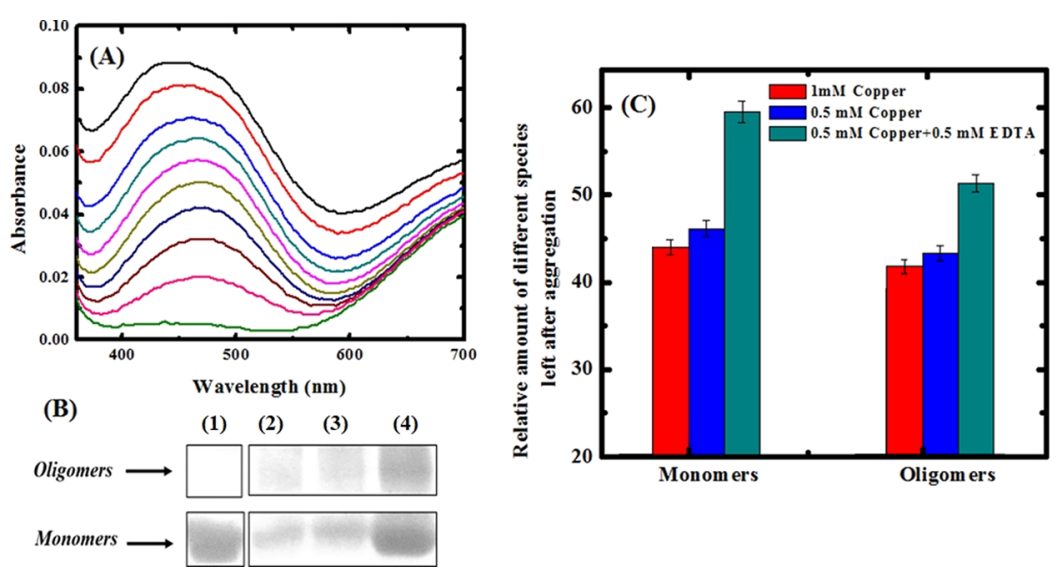

Figure 9. (A) UV spectra of DA in buffer $(20 \mathrm{mM}$ Tris, $0.1 \mathrm{M} \mathrm{NaCl}, \mathrm{pH} 7.5)$ in the presence of copper and EDTA. (B) SDS-PAGE of $\alpha$-synuclein after its aggregation in DA in the presence of $1 \mathrm{mM}$ copper (lane 2), $0.5 \mathrm{mM}$ copper (lane 3), and $0.5 \mathrm{mM}$ copper + 0.5 mM EDTA (lane 4). Lane 1 shows the monomeric protein before aggregation. (C) Quantification of the amount of monomers and oligomers as detected in the SDS-PAGE in (B).

interference in the usual pathway by the external agents (here, $\alpha$-synuclein or glycerol). In our case, we speculate that both glycerol and $\alpha$-synuclein interact with the oxidation products of DA.

DA Forms Widely Different Oxidation Products in the Presence of Copper and Exhibits Linear Kinetics. In the presence of copper, the spectrum of DA was remarkably sharper (as compared to buffer alone) and showed a significant red shift with increasing time (Figure 8A). This is in contrast to the blue-shifted peak or the red-shifted shoulder observed in the absence of copper (Figure 7A). A peak of lower intensity at $650 \mathrm{~nm}$ could also be observed in these spectra, which corresponded to the copper-water complex. It can be seen that the kinetics of oxidation of DA was linear in the presence of copper (Figure $8 \mathrm{~B}$, square), whereas it was exponential in its absence (Figure 7B,C). Moreover, the absence of new peaks at 405 or $475 \mathrm{~nm}$ indicates that the common oxidation products of DA such as dopaminoquinone or dopaminochrome are either not formed to any significant extent in the presence of copper or are involved in complexation with copper. The IR spectra of DA also exhibited a significant change in the presence of copper (Figure S4), suggesting that the metal drastically affects the prominent vibrations in the molecule. The different oxidation products formed in the presence of copper or the changes brought about in DA because of the presence of copper may have a close connection with the suppressed formation of the SDS-resistant oligomers in copper-DA.

Plausible Mechanism of Inhibition of Oligomerization in the Presence of Copper. The oxidation of DA via a large number of intermediates such as dopaminochromes, dopaminoquinone, or dihydroxyindole is accompanied by the simultaneous liberation of reactive oxygen species such as superoxides and peroxides. ${ }^{3,26}$ Although copper catalyzes the oxidation of DA, it does so in terms of the formation of hydrogen peroxide. In the presence of copper, DA takes a different route altogether for its oxidation, wherein the formation of common oxidation products such as dopaminochrome or dopaminoquinone are significantly suppressed, as discussed previously. Most importantly, DA instantaneously forms a complex with copper to form a copper catecholate-type complex-a step which will be absent in the absence of copper. $^{26,27}$ The formation of copper-DA complex is also a major route for the elimination of DA from the medium, thereby preventing the oxidation reaction to proceed further. Conway et al. had screened a number of compounds and had inferred that the presence of catechol moiety was essential in the prevention of the fibrillation of $\alpha$-synuclein. As the catechol moiety of DA is involved in complexation with copper, the former is not able to interact with $\alpha$-synuclein, because of which no adduct with the protein is formed in the presence of DA and copper (Figure 6A), and hence, no SDS-resistant oligomers are formed. Using the mechanism provided by Pham et al., ${ }^{26}$ a comparison of the routes of the DA mediated aggregation of $\alpha$-synuclein in the absence and presence of copper is presented in Scheme 2.

In Scheme 2, $\mathrm{H}_{2} \mathrm{~A}$ is the leukoaminochrome, $\mathrm{Q}$ is the quinone, while $\mathrm{A}$ is the aminochrome. It has been suggested that the copper-DA complex disintegrates via an electrontransfer process between the metal center and DA. The 
electron transfer, and the subsequent reduction and oxidation of $\mathrm{Cu}(\mathrm{II})$ and $\mathrm{Cu}(\mathrm{I})$, respectively, becomes the predominant reactions in the decomposition, as opposed to the formation of dopaminochrome and dopaminoquinone. ${ }^{26}$ Even the formation of dopaminoquinone in the presence of the metal requires the oxidized state $(\mathrm{Cu}(\mathrm{II}))$ of the metal. However, as already mentioned, the metal center is continuously undergoing an exchange between the oxidized and reduced state, with the oxidized state being less populated. ${ }^{26}$ As a result, such intermediates do not get formed to a large extent. Even so, the minute amount of oxidation products such as semiquinone that are formed are also capable of complexation with copper. $^{28}$ As a result, these intermediates too, if any, do not get a chance to bind to the oligomeric species in the aggregation pathway, thereby inhibiting the formation of SDSresistant oligomers in the copper-DA system.

Suppressing the oxidation of DA has been drastically found to reduce the presence of SDS-resistant oligomers. Pham et al. ${ }^{3}$ have shown that the formation of SDS-resistant oligomers may be completely inhibited even in the presence of DA if antioxidants are present in the medium. The formation of SDSresistant oligomers is inhibited in the presence of copper, not because of inhibition of oxidation but because of the complexation of DA and its oxidation products with copper. These two independent observations prove to be a good support for the fact that prevention/modification of the oxidation of DA from its intrinsic pathway can help in overcoming the formation of SDS-resistant oligomers.

Sequestration of Copper lons Reverts the Oxidation Pathway of DA to Its Usual Route. In order to clarify whether our speculation regarding the complexation of copper with DA is appropriate or not, the oxidation of the copper-DA system was studied in the presence of ethylenediaminetetraacetic acid (EDTA). The UV-visible spectra in the presence of EDTA were found to be similar to those observed in buffer alone and were blue-shifted with time (Figure 9A). This is because of the sequestration of copper by EDTA, which is indicated by the shift in the peak of the copper complex from $650 \mathrm{~nm}$ (in buffer) to nearly $700 \mathrm{~nm}$ (in EDTA). The complexation of copper with EDTA prevented the interaction of copper with DA, and hence, the oxidation proceeded in its usual route (similar to that in DA alone). In order to see whether the sequestration of copper had a similar effect on the aggregation of $\alpha$-synuclein, the aggregation was carried out in the presence of DA, copper, and EDTA. The combination resulted in an increase of the SDS-resistant oligomers (Figure 9B) by about $18 \%$ (Figure 9C), suggesting that the sequestration of copper by EDTA does not allow the copper-DA complex to be formed. As a result, DA oxidizes itself in its usual manner forming dopaminochrome and dopaminoquinones, which further interacts with the protein to form the SDS-resistant oligomers. The quantification of the amount of monomers and oligomers from SDS-PAGE is presented in Figure 9C. Thus, our speculation that the modified oxidation pathway of DA in the presence of copper is responsible for the diminished formation of SDS-resistant oligomers seems correct.

Copper Stabilizes the Aggregates in the Presence of DA, Whereas Glycerol Destabilizes Them. In order to check whether copper and glycerol were effective in preventing the dissolution of the fibrils by DA or not, the aggregation was first carried out with and without copper or glycerol (in the absence of DA), and DA was added to them after $24 \mathrm{~h}$ of aggregation. DA is already known to disintegrate preformed fibrils (formed in buffer alone) into SDS-resistant oligomers, and this can also be confirmed from SDS-PAGE (Figure 10,

\section{(1) (2) (3)}

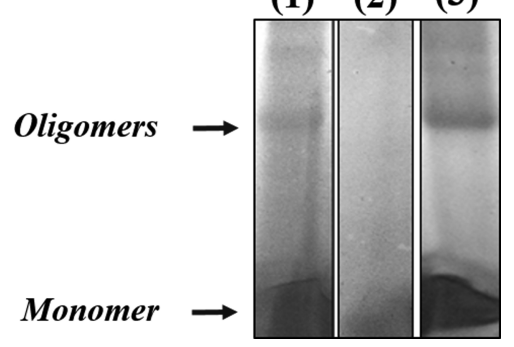

Figure 10. SDS-PAGE of the DA-induced disaggregation of the fibrils formed in buffer alone (lane 1), copper, (lane 2), and glycerol (lane 3).

lane 1). However, the affinity of copper and DA toward each other is so strong that the presence of the former does not allow the latter to interact with preformed fibrils (in copper) to disintegrate them. As a result, the SDS-PAGE did not show any traces of oligomers or monomers, suggesting that the fibrils formed in copper remained intact even in the presence of DA (Figure 10, lane 2). On the other hand, the addition of DA to the fibrils formed in glycerol led to the noticeable formation of the SDS-resistant oligomers (Figure 10, lane 3). The overall behavior can be described as shown in Scheme 3 .

Scheme 3. Behavior of DA during the Aggregation of $\alpha$ Synuclein in the Presence of Glycerol/Copper

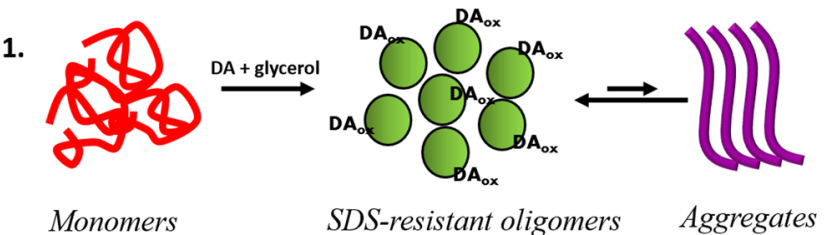

2.

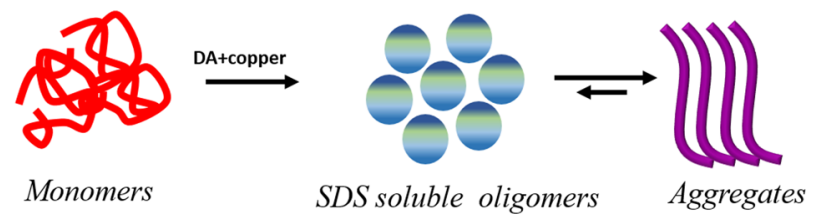

The overall study can be summarized as shown in Scheme 4 . Scheme 4 represents five different conditions of aggregation, numbered from 1 through 5 . The first condition (1) represents the situation when $\alpha$-synuclein is aggregated in the presence of buffer alone. In buffer alone, $\alpha$-synuclein initially builds up an oligomeric population (multicolored spheres), which further proceeds to form the insoluble fibrils. These aspects are already known from the previous studies and are discussed only briefly here. The second condition (2) shows the pathway of aggregation in the presence of DA. DA and its oxidation products bind to $\alpha$-synuclein via various modifications of the amino acid residues as discussed previously and lead to the formation of SDS-resistant oligomers (green spheres). These interactions prevent the other interactions that are necessary to form fibrils. The interactions of DA and its oxidation products with the fibrils are strong enough to even disintegrate the fibrils. This is shown by an arrow between routes (1) and (2). 
Scheme 4. Overall Aggregation Pathway of $\alpha$-Synuclein in the Presence of Different Additives or Combination of Additives

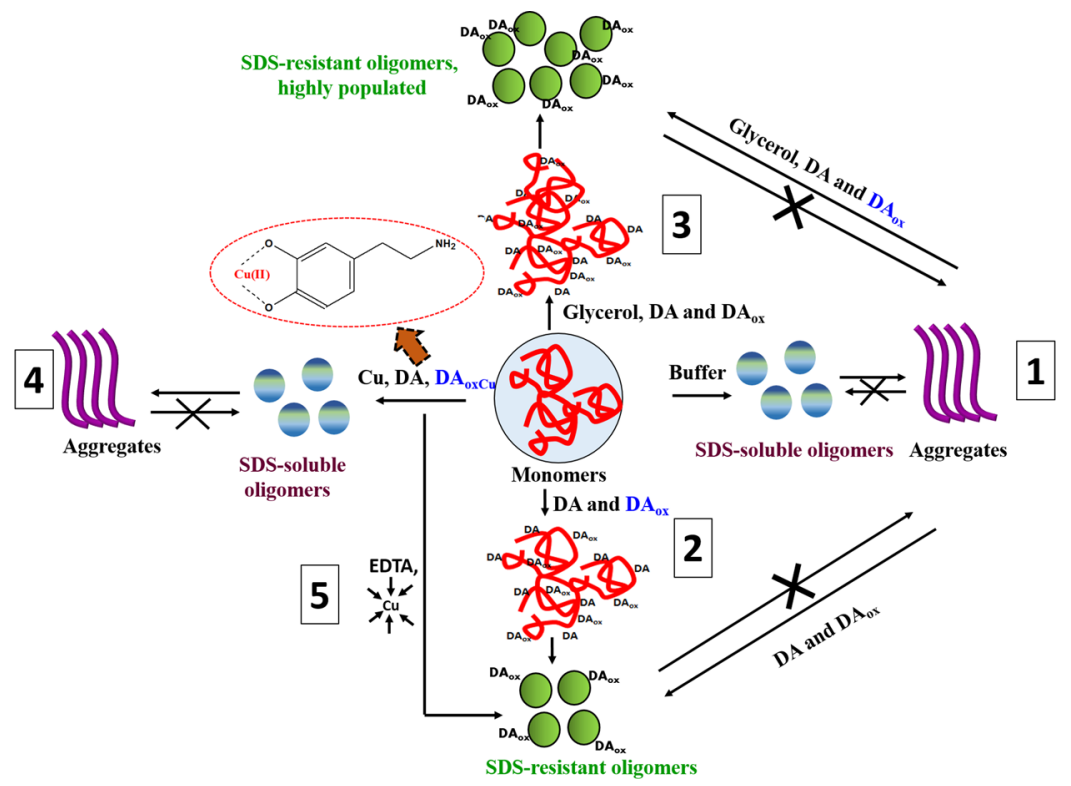

(A)
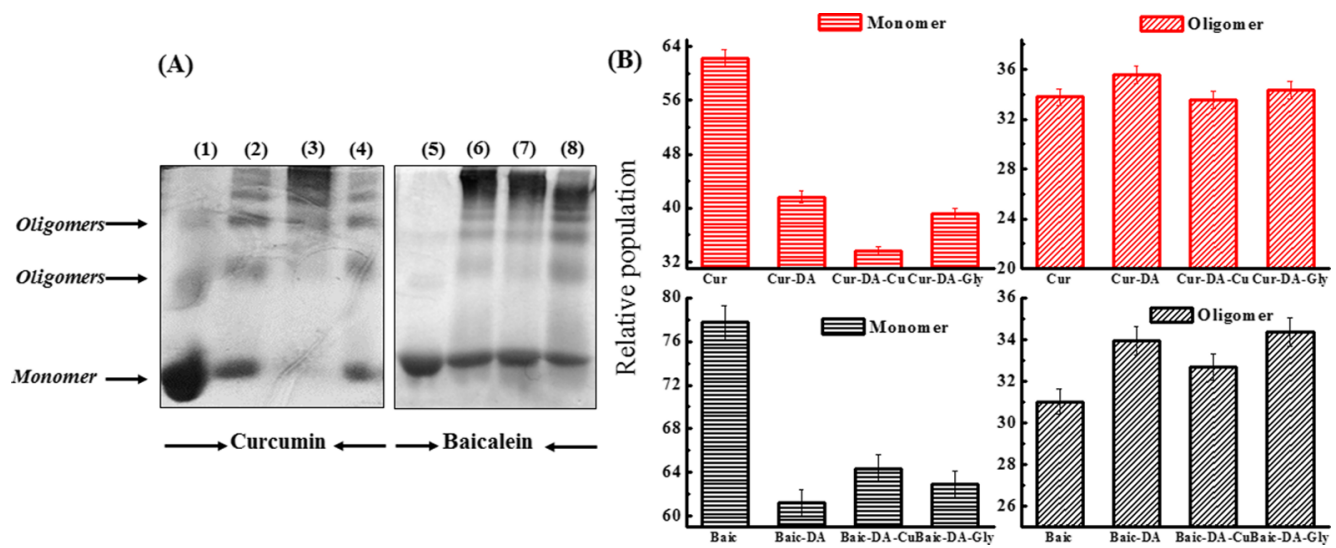

Figure 11. (A) Effect of $100 \mu \mathrm{M}$ of curcumin (Cur) and $100 \mu \mathrm{M}$ of baicalein (Baic) on the DA-mediated aggregation of $\alpha$-synuclein in the presence of different additives. The concentrations of copper and glycerol are $0.1 \mathrm{mM}$ and $2 \mathrm{M}$, respectively. (B) Quantification of monomers and oligomers from SDS-PAGE in (A) for the different combinations of additives.

The third scenario (3) is the aggregation in the presence of DA and glycerol. The overall pathway is same as that in DA alone, expect for the fact that glycerol increases the formation of SDSresistant oligomers (green spheres) and also increases the interaction of DA and its oxidation product with the side chains of the protein. As a result, the degradation of fibrils in the presence of glycerol also results in an increased amount of the SDS-resistant oligomers, as compared to that in DA alone. This is shown by an arrow between routes (1) and (3). The fourth scenario (4) represents the aggregation in the presence of copper and DA. The overall pathway is different from that observed in DA (or glycerol-DA), but similar to that in buffer alone [route (1)]. Oligomers formed in the presence of copper and DA are soluble in SDS, unlike those observed in route (2) or (3). The effect of DA on the protein is largely overpowered by the strong kinetics of the complexation between copper and DA. As a result of the complexation, DA does not interact with the protein and hence do not form SDS-resistant oligomers. That the presence of copper does not let DA act on the protein is also clarified from the absence of any fibril disintegration in the presence of copper and DA (Figure 10). In order to further confirm that complexation was indeed the reason behind the unresponsive behavior of DA in the presence of copper, the aggregation was carried out in the presence of copper, EDTA, and DA (route 5). Indeed, the addition of EDTA reverted the route back to the formation of SDS-resistant oligomers. Effectively, any strategy that can prevent the formation of the protein-DA adduct can prevent the formation of the SDSresistant oligomers.

Effect of Curcumin and Baicalein on Aggregation in DA, Copper-DA, and Glycerol-DA. It was worth examining whether the effect imparted by copper or glycerol was universal or not, and this was confirmed using polyphenols. Polyphenols are widely known to modulate the aggregation of many proteins, including $\alpha$-synuclein, and this class of compounds is being largely considered from the view point of drugs for many neurodegenerative diseases. Therefore, the aggregation of $\alpha$-synuclein was carried out with baicalein and curcumin, in the presence of DA, copper-DA, and glycerol-DA. Baicalein has been already shown to promote the oligomerization of $\alpha$-synuclein from its monomeric as well as fibrillar form. ${ }^{29,30}$ Curcumin on the other hand is known to 
prevent both the oligomerization and fibrillation of $\alpha$ synuclein. $^{22,31}$ The aggregation was studied using SDSPAGE, and the gels were quantified to get an estimate of the effect of these polyphenols. Unlike in the absence of any additive, curcumin was capable of forming some SDS-resistant oligomers, while such oligomers were quite insignificant in baicalein (Figure 11A, lanes 1 and 5). DA increased the formation of these SDS-resistant oligomers in curcumin by $5.2 \%$, whereas in the presence of baicalein, these oligomers increased up to $9.4 \%$ (Figure 11A, lanes 2 and 6). However, the oligomers were lesser in baicalein-DA system than in the curcumin-DA system. Copper, in the presence of DA, was indeed able to prevent the formation of SDS-resistant oligomers of $\alpha$-synuclein in both baicalein and curcumin (Figure 11A, lane 3 and 7). Glycerol was able to decrease the DA-induced oligomers in curcumin by $3.4 \%$, whereas such oligomers were promoted by glycerol in baicalein by $1.3 \%$. Nevertheless, the monomeric content was far lesser in curcumin-DA system than in the baicalein-DA system for all the conditions tested. The quantification of the gel is presented in Figure 11B. All the observations are summarized in Table 1 in order to provide a better picture of the effect of

Table 1. Presence of Oligomers and Monomers/SDSSoluble Species in the Presence of Different Additives

\begin{tabular}{|c|c|c|}
\hline system & $\begin{array}{l}\text { SDS-resistant } \\
\text { oligomers }\end{array}$ & $\begin{array}{l}\text { monomers/SDS-soluble } \\
\text { species }\end{array}$ \\
\hline no additive & $-\mathrm{ve}$ & +ve \\
\hline DA & + ve & $+\mathrm{ve}$ \\
\hline DA-Gly & +ve (high) & + ve \\
\hline DA-copper & -ve (very low) & +ve (low) \\
\hline curcumin & +ve & +ve (high) \\
\hline DA-curcumin & +ve (high) & +ve (low) \\
\hline DA-curcumin-Gly & +ve (nearly same) & +ve (low) \\
\hline $\mathrm{DA}-$ curcumin $-\mathrm{Cu}$ & -ve (very low) & +ve (low) \\
\hline baicalein & +ve (very low) & +ve (high) \\
\hline DA-baicalein & +ve (low) & +ve (high) \\
\hline DA-baicalein-Gly & +ve (high) & +ve (low) \\
\hline $\mathrm{DA}-$ baicalein-Cu & -ve (very low) & +ve (high) \\
\hline
\end{tabular}

different additives. To summarize, it can be concluded that copper can still act to reduce the population of the SDSresistant oligomers that are formed in DA in the presence of curcumin or baicalein.

\section{CONCLUSIONS}

The study shows that the commonly known enhancers/ inhibitors of protein aggregation may behave differently under some cases. For the case of this natively unfolded protein, both copper and glycerol could expedite the onset of aggregation in buffer alone, but one of them enhanced the extent of aggregation, whereas the other decreased the same. During the aggregation in DA, no SDS-resistant oligomers could be detected in copper, whereas such oligomers were found to be promoted in the presence of glycerol.

It is known that inhibition of fibrillation is not the only process that needs to be considered in an aggregation process. Glycerol is a well-known stabilizer of proteins, and for the particular case of $\alpha$-synuclein too, glycerol was able to reduce the extent of fibrillation. Copper, on the other hand, enhanced the fibrillation of the protein. In a closer physiological scenario, where DA is also present, glycerol was found to promote the formation of SDS-resistant oligomers, whereas copper reduced the same. This is because DA is not able to assist in the formation of SDS-resistant oligomers in the presence of copper, thus driving the aggregation toward the less harmful fibrils. Glycerol, on the other hand, blocks the aggregation at the more detrimental oligomeric state promoted by DA. Our study reveals a very crucial aspect. Successful attempts to suppress the aggregation of $\alpha$-synuclein, particularly by the use of additives/drugs, might indeed be a step toward curbing PD; however, it is equally important to check whether those additives still respond in the same manner even in an environment which has DA. This is because of the fact that $\alpha$-synuclein and DA are tightly regulated in the presynaptic terminal, and the response of an additive toward the aggregation of $\alpha$-synuclein might be drastically inversed in the presence of DA.

\section{MATERIALS AND METHODS}

Materials. The plasmid of $\alpha$-synuclein was received as a kind gift from Dr. Monica Sundd, National Institute of Immunology, New Delhi, India. Tris buffer and copper sulfate pentahydrate were purchased from Merck. ThT, DA hydrochloride, curcumin, and baicalein were purchased from SigmaAldrich. Glycerol was purchased from SD Fine Chemicals, India, whereas SDS was purchased from Sisco Research Laboratory, India. All the solutions, except otherwise stated, were prepared in $20 \mathrm{mM}$ Tris, $\mathrm{pH} 7.5$, and $0.1 \mathrm{M} \mathrm{NaCl}$. Stock solutions of curcumin and baicalein were prepared in $100 \%$ ethanol procured from Merck.

Protein Expression and Purification. The Escherichia coli strain BL21-DE3 was used for the expression of human $\alpha$ synuclein, whose gene is encoded in the pET 21 a vector. The expression of the protein was induced by the addition of $1 \mathrm{mM}$ IPTG for $4 \mathrm{~h}$, once the optical density of the bacterial culture was 0.6 at $600 \mathrm{~nm}$. The harvested cells were dissolved and lysed in $20 \mathrm{mM}$ Tris, $0.1 \mathrm{M} \mathrm{NaCl}, \mathrm{pH}$ 7.5. The protein was isolated using the $\mathrm{pH}$ precipitation method and then further purified using a $\mathrm{NaCl}$ gradient on a Source $\mathrm{Q}$ column attached to a GE AKTA purifier FPLC system. The purity of the protein was checked using SDS-PAGE.

UV-Visible Spectroscopy. The concentration of the protein was checked using a molar extinction coefficient of $5600 \mathrm{M}^{-1} \mathrm{~cm}^{-1}$ at $276 \mathrm{~nm}$ in a Shimadzu $2600 \mathrm{UV}-$ visible spectrophotometer. The concentration of ThT was checked using a molar extinction coefficient of $35000 \mathrm{M}^{-1} \mathrm{~cm}^{-1}$ at 412 $\mathrm{nm}$. For the absorption studies of DA, the compound was dissolved in the required additives immediately before taking the measurement.

Aggregation Assay. $\alpha$-Synuclein at the required concentration was prepared in the absence and presence of additives and put into aggregation at $37^{\circ} \mathrm{C}$ in an incubator, with shaking at $250 \mathrm{rpm}$. Aliquots were collected at the required intervals and diluted with $20 \mathrm{mM}$ Tris buffer. ThT was added to it, and the ThT fluorescence was checked at $482 \mathrm{~nm}$, upon excitation at $450 \mathrm{~nm}$ in a Varian Cary Eclipse fluorescence spectrophotometer.

Fluorescence Microscopy. The aggregated solutions (2 $\mu \mathrm{L}$ ) were diluted in 1:1 ratio, and ThT was added to it. The samples $(4 \mu \mathrm{L})$ were put on a glass slide and allowed to dry. The samples were then viewed using a Nikon Eclipse $\mathrm{Ni}$ H600L fluorescence microscope.

Transmission Electron Microscopy. The aggregated solution of $\alpha$-synuclein $(2 \mu \mathrm{L})$ in the presence of additives 
was put on a copper grid. The excess solution was soaked, and the grid was stained with uranyl acetate solution. This was allowed to dry for $30 \mathrm{~min}$ and then viewed with an FEI Tecnai TF20 transmission electron microscope.

Circular Dichroism. The supernatant left after the aggregation in the presence of different additives or a combination of additives was separated by centrifuging the aggregated solution at $12000 \mathrm{rpm}$. The supernatant was diluted 10-folds, and the circular dichroism (CD) spectrum was collected in an AVIV Sx-420 CD spectrophotometer. The resultant spectra are an average of five scans.

Size Exclusion Chromatography. The aggregated samples of $200 \mu \mathrm{M} \alpha$-synuclein $(1 \mathrm{~mL})$ in DA in the presence and absence of additives was centrifuged at a high speed to separate out any insoluble particles. The supernatant was collected and loaded onto a Superdex 200 increase 10/300 GL size exclusion column connected to a GE AKTA purifier FPLC system using a $1 \mathrm{~mL}$ loop, injecting $800 \mu \mathrm{L}$ of the sample into the column. The elution was carried out isocratically using 20 $\mathrm{mM}$ Tris buffer, $0.1 \mathrm{M} \mathrm{NaCl}, \mathrm{pH}$ 7.5. The absorbance from the sample at 280 and $215 \mathrm{~nm}$ was recorded.

\section{ASSOCIATED CONTENT}

\section{S Supporting Information}

The Supporting Information is available free of charge on the ACS Publications website at DOI: 10.1021/acsomega.8b00993.

Fluorescence spectra of DA in the presence of $\alpha$ synuclein under quiescent condition, final appearance of the DA solution after 3 days of incubation, change in the absorbance spectrum of DA during its oxidation in the presence of glycerol, and IR spectra of DA in the presence of buffer alone and copper at two time points (PDF)

\section{AUTHOR INFORMATION}

\section{Corresponding Author}

*E-mail: sdeep@chemistry.iitd.ac.in (S.D.).

\section{ORCID}

Shashank Deep: 0000-0002-4908-8205

\section{Present Address}

${ }^{\dagger}$ University of North Carolina, Department of Biochemistry and Biophysics, Chapel Hill, NC 27599, USA.

\section{Notes}

The authors declare no competing financial interest.

\section{ACKNOWLEDGMENTS}

S.D. thanks the Council of Scientific and Industrial Research (CSIR), India, for the generous funding to carry out the above research. S.S. thanks the CSIR, Government of India, for the fellowship. M.A.I.K. thanks the UGC, Government of India, for the fellowship. S.S. thanks Sandeep Kumar Rai, Department of Chemistry, IIT-Delhi, for his help during the revision of the manuscript. S.S. thanks Dr. Pramit Kumar Choudhury, Department of Chemistry, IIT-Delhi, for the usage of the UV-visible spectrophotometer.

\section{REFERENCES}

(1) Fahn, S.; Sulzer, D. Neurodegeneration and Neuroprotection in Parkinson Disease. NeuroRx 2004, 1, 139-154.
(2) Li, J.; Zhu, M.; Manning-Bog, A. B.; Di Monte, D. A.; Fink, A. L. Dopamine and L-dopa disaggregate amyloid fibrils: implications for Parkinson's and Alzheimer's disease. Faseb. J. 2004, 18, 962-964.

(3) Pham, C. L. L.; Leong, S. L.; Ali, F. E.; Kenche, V. B.; Hill, A. F.; Gras, S. L.; Barnham, K. J.; Cappai, R. Dopamine and the Dopamine Oxidation Product 5,6-Dihydroxylindole Promote Distinct OnPathway and Off-Pathway Aggregation of $\alpha$-Synuclein in a $\mathrm{pH}$ Dependent Manner. J. Mol. Biol. 2009, 387, 771-785.

(4) Lee, H.-J.; Baek, S. M.; Ho, D.-H.; Suk, J.-E.; Cho, E.-D.; Lee, S.J. Dopamine promotes formation and secretion of non-fibrillar alphasynuclein oligomers. Exp. Mol. Med. 2011, 43, 216-222.

(5) Conway, K. A.; Rochet, J.-C.; Bieganski, R. M.; Lansbury, P. T., Jr. Kinetic Stabilization of the alpha -Synuclein Protofibril by a Dopamine-alpha -Synuclein Adduct. Science 2001, 294, 1346-1349.

(6) Leong, S. L.; Cappai, R.; Barnham, K. J.; Pham, C. L. L. Modulation of $\alpha$-Synuclein Aggregation by Dopamine: A Review. Neurochem. Res. 2009, 34, 1838-1846.

(7) Fink, A. L. The Aggregation and Fibrillation of $\alpha$-Synuclein. Acc. Chem. Res. 2006, 39, 628-634.

(8) Outeiro, T. F.; Klucken, J.; Bercury, K.; Tetzlaff, J.; Putcha, P.; Oliveira, L. M. A.; Quintas, A.; McLean, P. J.; Hyman, B. T. Dopamine-Induced Conformational Changes in Alpha-Synuclein. PloS One 2009, 4, No. e6906.

(9) Rochet, J.-C.; Outeiro, T. F.; Conway, K. A.; Ding, T. T.; Volles, M. J.; Lashuel, H. A.; Bieganski, R. M.; Lindquist, S. L.; Lansbury, P. T. Interactions Among $\alpha$-Synuclein, Dopamine, and Biomembranes: Some Clues for Understanding Neurodegeneration in Parkinson's Disease. J. Mol. Neurosci. 2004, 23, 023-034.

(10) Bisaglia, M.; Tosatto, L.; Munari, F.; Tessari, I.; de Laureto, P. P.; Mammi, S.; Bubacco, L. Dopamine quinones interact with $\alpha$ synuclein to form unstructured adducts. Biochem. Biophys. Res. Commun. 2010, 394, 424-428.

(11) Paik, S. R.; Shin, H.-J.; Lee, J.-H.; Chang, C.-S.; Kim, J. Copper(II)-induced self-oligomerization of $\alpha$-synuclein. Biochem. J. 1999, 340, 821-828.

(12) Uversky, V. N.; Li, J.; Fink, A. L. Metal-triggered Structural Transformations, Aggregation, and Fibrillation of Human $\alpha$ Synuclein. J. Biol. Chem. 2001, 276, 44284-44296.

(13) Rasia, R. M.; Bertoncini, C. W.; Marsh, D.; Hoyer, W.; Cherny, D.; Zweckstetter, M.; Griesinger, C.; Jovin, T. M.; Fernandez, C. O. Structural characterization of copper(II) binding to -synuclein: Insights into the bioinorganic chemistry of Parkinson's disease. Proc. Natl. Acad. Sci. U.S.A. 2005, 102, 4294-4299.

(14) Rose, F.; Hodak, M.; Bernholc, J. Mechanism of copper(II)induced misfolding of Parkinson's disease protein. Sci. Rep. 2011, 1, 11 .

(15) Vagenende, V.; Yap, M. G. S.; Trout, B. L. Mechanisms of protein stabilization and prevention of protein aggregation by glycerol. Biochemistry 2009, 48, 11084-11096.

(16) Gekko, K.; Timasheff, S. N. Mechanism of protein stabilization by glycerol: preferential hydration in glycerol-water mixtures. Biochemistry 1981, 20, 4667-4676.

(17) Saha, S.; Deep, S. Glycerol inhibits the primary pathways and transforms the secondary pathway of insulin aggregation. Phys. Chem. Chem. Phys. 2016, 18, 18934-18948.

(18) Shukla, D.; Schneider, C. P.; Trout, B. L. Molecular level insight into intra-solvent interaction effects on protein stability and aggregation. Adv. Drug Deliv. Rev. 2011, 63, 1074-1085.

(19) Brown, C. R.; Hong-Brown, L. Q.; Welch, W. J. Correcting temperature-sensitive protein folding defects. J. Clin. Invest. 1997, 99, $1432-1444$

(20) Yang, D.-S.; Yip, C. M.; Huang, T. H. J.; Chakrabartty, A.; Fraser, P. E. Manipulating the Amyloid- $\beta$ Aggregation Pathway with Chemical Chaperones. J. Biol. Chem. 1999, 274, 32970-32974.

(21) Cappai, R.; Leck, S.-L.; Tew, D. J.; Williamson, N. A.; Smith, D. P.; Galatis, D.; Sharples, R. A.; Curtain, C. C.; Ali, F. E.; Cherny, R. A.; Culvenor, J. G.; Bottomley, S. P.; Masters, C. L.; Barnham, K. J.; Hill, A. F. Dopamine promotes $\alpha$-synuclein aggregation into SDS- 
resistant soluble oligomers via a distinct folding pathway. Faseb. J. 2005, 19, 1377-1379.

(22) Ahmad, B.; Lapidus, L. J. Curcumin Prevents Aggregation in $\alpha$ Synuclein by Increasing Reconfiguration Rate. J. Biol. Chem. 2012, 287, 9193-9199.

(23) Norris, E. H.; Giasson, B. I.; Hodara, R.; Xu, S.; Trojanowski, J. Q.; Ischiropoulos, H.; Lee, V. M.-Y. Reversible Inhibition of $\alpha$ Synuclein Fibrillization by Dopaminochrome-mediated Conformational Alterations. J. Biol. Chem. 2005, 280, 21212-21219.

(24) Li, N.; Wang, H.-B.; Thia, L.; Wang, J.-Y.; Wang, X. Enzymaticreaction induced production of polydopamine nanoparticles for sensitive and visual sensing of urea. Analyst 2015, 140, 449-455.

(25) Klosterman, L.; Bettinger, C. J. Calcium-Mediated Control of Polydopamine Film Oxidation and Iron Chelation. Int. J. Mol. Sci. 2016, 18, 14.

(26) Pham, A. N.; Waite, T. D. Cu(II)-catalyzed oxidation of dopamine in aqueous solutions: mechanism and kinetics. J. Inorg. Biochem. 2014, 137, 74-84.

(27) Balla, J.; Kiss, T.; Jameson, R. F. Copper(II)-catalyzed oxidation of catechol by molecular oxygen in aqueous solution. Inorg. Chem. 1992, 31, 58-62.

(28) Thompson, J. S.; Calabrese, J. C. Copper-catechol chemistry. Synthesis, spectroscopy, and structure of bis(3,5-di-tert-butyl-osemiquinato)copper(II). J. Am. Chem. Soc. 1986, 108, 1903-1907.

(29) Zhu, M.; Rajamani, S.; Kaylor, J.; Han, S.; Zhou, F.; Fink, A. L. The Flavonoid Baicalein Inhibits Fibrillation of $\alpha$-Synuclein and Disaggregates Existing Fibrils. J. Biol. Chem. 2004, 279, 26846-26857. (30) Hong, D.-P.; Fink, A. L.; Uversky, V. N. Structural Characteristics of $\alpha$-Synuclein Oligomers Stabilized by the Flavonoid Baicalein. J. Mol. Biol. 2008, 383, 214-223.

(31) Singh, P. K.; Kotia, V.; Ghosh, D.; Mohite, G. M.; Kumar, A.; Maji, S. K. Curcumin Modulates $\alpha$-Synuclein Aggregation and Toxicity. ACS Chem. Neurosci. 2013, 4, 393-407. 